\title{
Digital simulation of an arbitrary stationary stochastic process by spectral representation
}

\section{Yura, Harold T.; Hanson, Steen Grüner}

Published in:

Journal of the Optical Society of America A

Link to article, DOI:

10.1364/JOSAA.28.000675

Publication date:

2011

Document Version

Publisher's PDF, also known as Version of record

Link back to DTU Orbit

Citation (APA):

Yura, H. T., \& Hanson, S. G. (2011). Digital simulation of an arbitrary stationary stochastic process by spectral representation. Journal of the Optical Society of America A, 28(4), 675-685.

https://doi.org/10.1364/JOSAA.28.000675

\section{General rights}

Copyright and moral rights for the publications made accessible in the public portal are retained by the authors and/or other copyright owners and it is a condition of accessing publications that users recognise and abide by the legal requirements associated with these rights.

- Users may download and print one copy of any publication from the public portal for the purpose of private study or research.

- You may not further distribute the material or use it for any profit-making activity or commercial gain

- You may freely distribute the URL identifying the publication in the public portal

If you believe that this document breaches copyright please contact us providing details, and we will remove access to the work immediately and investigate your claim. 


\title{
Digital simulation of an arbitrary stationary stochastic process by spectral representation
}

\author{
Harold T. Yura ${ }^{1}$ and Steen G. Hanson ${ }^{2, *}$ \\ ${ }^{1}$ Electronics and Photonics Laboratory, The Aerospace Corporation, Los Angeles California 90009, USA \\ ${ }^{2}$ DTU Fotonik, Department of Photonics Engineering, Danish Technical University, 4000 Roskilde, Denmark \\ ${ }^{*}$ Corresponding author: vsgh@fotonik.dtu.dk
}

Received October 12, 2010; revised January 14, 2011; accepted January 17, 2011; posted February 3, 2011 (Doc. ID 136501); published March 31, 2011

\begin{abstract}
In this paper we present a straightforward, efficient, and computationally fast method for creating a large number of discrete samples with an arbitrary given probability density function and a specified spectral content. The method relies on initially transforming a white noise sample set of random Gaussian distributed numbers into a corresponding set with the desired spectral distribution, after which this colored Gaussian probability distribution is transformed via an inverse transform into the desired probability distribution. In contrast to previous work, where the analyses were limited to auto regressive and or iterative techniques to obtain satisfactory results, we find that a single application of the inverse transform method yields satisfactory results for a wide class of arbitrary probability distributions. Although a single application of the inverse transform technique does not conserve the power spectra exactly, it yields highly accurate numerical results for a wide range of probability distributions and target power spectra that are sufficient for system simulation purposes and can thus be regarded as an accurate engineering approximation, which can be used for wide range of practical applications. A sufficiency condition is presented regarding the range of parameter values where a single application of the inverse transform method yields satisfactory agreement between the simulated and target power spectra, and a series of examples relevant for the optics community are presented and discussed. Outside this parameter range the agreement gracefully degrades but does not distort in shape. Although we demonstrate the method here focusing on stationary random processes, we see no reason why the method could not be extended to simulate non-stationary random processes. (C) 2011 Optical Society of America
\end{abstract}

OCIS codes: $\quad 030.1640,120.7250,030.1670,030.6140,030.6600$.

\section{INTRODUCTION}

The purpose of this paper is to present a straightforward, efficient, and computationally fast method by which a large number of discrete samples of a wide class of stationary random processes with both specified power spectral densities (PSDs) and an arbitrary specified probability distribution function can be accurately generated. The examples illustrated here will primarily address the optics community. In the study of (complex) physical systems involving stationary random processes, the relationship between the input and the system output is often desired to model the system response. In the past several decades, simulation methods in various fields of science and engineering have been developed to take advantage of modern computational advancements (e.g., telecommunications, radar and signal processing, speckle phenomena, optical propagation through the atmosphere, wind engineering, and structural mechanics). In general, to model system response, it is highly desirable to employ simulation techniques that accurately reflect both the underlying probability distribution and PSD, which are usually given by physical theories and/or by spectral analysis of measured data.

Although, as discussed below, the simulation of a colored Gaussian process with a given PSD is well established, the corresponding modeling of an arbitrary colored non-Gaussian process, primarily due to rigorous mathematical difficulties, has not yet achieved as much widespread attention. As alluded to in Section 2, in the engineering mechanics literature, an iterative procedure has been developed for zero mean probability distributions that first generates Gaussian sample fields and then maps them into corresponding non-Gaussian samples [1]. The authors of [1] claim that, because their inverse transformation mapping technique is nonlinear, an iterative procedure must be used in order to obtain satisfactory agreement between the simulated and target function's PSDs. Strictly speaking, the inverse transformation mapping used in [1] is nonlinear, however it is, so to speak, weakly nonlinear in that, in many cases of practical concern, no iterative procedure is required to obtain accurate results for both the desired probability distribution and the PSD. Although a single application of the inverse transform technique does not conserve the PSD exactly, it yields highly accurate numerical results for a wide range of probability distributions and target PSDs that are sufficient for system simulation purposes, and can thus be regarded as an accurate engineering approximation. As shown here, with modern digital computers, this procedure is simple to implement and computationally fast (for all cases considered here, more than $10^{5}$ colored sample data points were obtained in less than 1 min on a $2 \mathrm{GHz}$ Intel Core 2 Duo processor, which is by no means the fastest processor presently commercially available). Apparently, this mapping technique is not well known to the optics community and it is our intent to indicate its usefulness and viability as a simulation tool for a wide range of practical applications. In any case, if more accurate results are desired, one can implement the iterative process outlined in [1] . An extension of the above technique to two or more dimensions is straightforward, and is not addressed here. 
More recently, however, an algorithm for generating spectrally colored, non-Gaussian signals has been presented [2]. The approach is to use an iterative Fourier transform method to create a Gaussian random process with the appropriate spectral density and then apply a memoryless nonlinear transformation to achieve the desired probability density function (PDF), where the nonlinearity is approximated by a rankreordering procedure. In general, this transformation is not specified a priori, but, rather, is simulated via an iterative shuffling procedure. The authors of [2] reject the inverse cumulative distribution function (CDF) method, primarily because the inverse CDF is, in some cases, not analytically invertible. However, we illustrate below how one obtains excellent results by using Mathematica's efficient built in nonlinear curve fitting program. The noniterative inverse CDF method presented here is much easier for both implementing and simulating accurate colored non-Gaussian random processes for a wide variety of PDFs of interest in practice. The straightforward noniterative inverse CDF method, as implemented here, is based on generating a white noise (noncolored) sample of a Gaussian distribution, which is easily obtained from many computer programs, such as Mathematica, MATLAB, and Excel. This is in contrast to the method used in [2], where, as noted therein, "Although there are certain combinations of probability distribution and spectral density that are difficult to replicate, the approach is capable of faithfully generating a wide variety of colored, non-Gaussian signals. The algorithm also relies on the practitioners' ability to generate samples from the target probability distribution. In the instance where standard methods are not applicable, we have suggested using simple Monte Carlo rejection sampling technique to provide the needed sample."

Of course, it is not possible to check the accuracy of the resulting simulations for all possible PDFs and PSDs, and the interested user of the technique presented here should use the method described here to see if this method is viable for any given case of interest. In this regard, we note, for an arbitrary PDF, we present in Appendix B a general "rule of thumb" to obtain the parameter range where a single (i.e., noniterative) application of the inverse CDF method yields satisfactory results. Outside this parameter range of applicability, the agreement between the simulated and target PSDs gracefully degrades but does not significantly distort in shape.

Although, the simulations presented here were performed using the Mathematica computer program, similar results can be obtained using other computationally intensive programs, such as MATLAB.

This paper is organized as follows. In Section 2 we review briefly the development of simulating non-Gaussian processes, and introduce the inverse transform method and illustrate its usefulness by comparing the iterative results discussed in $[\underline{1}, \underline{2}]$ with a single application of the inverse transform method. In Section 3 we present simulation results for a wide class of probability distributions and PSDs of interest to the optics community. As indicated, the discrete sample distribution is in accord with the target probability distribution and its PSD is a satisfactory approximation to that of the target. Finally, in Section $\underline{4}$, we discuss our method and results and present some concluding remarks, while in Appendix $\underline{B}$ we derive a sufficiency expression that ascertains the parameter range where a single application of the inverse CDF method yields a satisfactory approximation to both the target PFD and PSD.

\section{SAMPLE GENERATION OF AN ARBITRARY STATIONARY RANDOM PROCESS}

Consider an arbitrary (given) continuous stationary random process, $X(t)$, where $t$ denotes time and is characterized by a PDF $p(x)$ [or, equivalently, by the cumulative distribution function (CDF) $F(x)$ ]. Although here we choose to illustrate the simulation method by considering random processes, similar results can also be obtained for stochastic random fields (e.g., speckle intensity in an observation plane). In the following, $p(x)$ and $F(x)$ are referred to as the "target PDF" and "target CDF," respectively. The technique we use here to generate discrete samples of $X$ is a Monte Carlo type simulation based on the so-called "inverse CDF transform method." Assume that a procedure is available for generating independent samples (i.e., white noise) of a random variable $Y$ with $\mathrm{CDF} F_{Y}$. Denoting random variables by upper case letters and the value it assumes by lower case letters [i.e., $\operatorname{PDF} p_{X}(x)$ ], then it can be shown that the random variable

$$
Z=F_{X}^{-1}\left(F_{Y}[y]\right)
$$

where $F_{X}^{-1}$ is the inverse target $\mathrm{CDF}$, has the distribution $F_{X}(x)$. Because both $F_{X}$ and $F_{Y}$ are bijective functions over the interval $\{0,1\}$, it follows that every $y$ in $Y$ is mapped to exactly one $x$ in $Z$, and it follows from this definition that no unmapped element exists in either $Z$ or $Y$. The distribution of $Z$ is therefore

$$
\begin{aligned}
F_{Z}(z) & =\operatorname{Prob}(Z \leq z)=\operatorname{Prob}\left[F_{X}^{-1}\left(F_{Y}[y]\right) \leq z\right] \\
& =\operatorname{Prob}\left[F(y)_{Y} \leq F_{X}(z)\right]=\operatorname{Prob}\left[y \leq F_{Y}^{-1}\left(F_{X}[z]\right)\right] \\
& =\mathrm{F}_{Y}\left[F_{Y}^{-1}\left(F_{X}[z]\right)\right]=F_{X}(x),
\end{aligned}
$$

so that $Z$ and $X$ have identical distributions. In the following, for brevity in notation, we omit the subscripts on the distributions under consideration.

Therefore, target distribution samples of $Z$ can be generated from samples of $Y$ transformed according to Eq. (2.1). The major advantage of this type of Monte Carlo simulation is that accurate results can be readily obtained for any stationary random process whose distribution function is known either analytically or numerically. In this regard, it is noted that both the Mathematica computer program [3] that is used here to obtain numerical results in a very timely manner and MATLAB support analytically a large number of probability distributions and their inverses, including those of interest to physical optics and optical and microwave propagation phenomena. Additionally, for cases where the distribution function is not known analytically, numerical results, as illustrated below, can be obtained very accurately from many mathematically efficient built-in nonlinear curve fitting computer programs. In the past, the only disadvantage of Monte Carlo simulation was that it was usually time consuming to obtain a large number of samples. However, as alluded to above, using mathematically efficient computer programs and present day digital computers, one can obtain a very large 
number of samples (typically $>10^{5}$ ) for times of the order of $1 \mathrm{~min}$ or less.

Examination of Eq. (2.1) reveals that, in general, any distribution can be uniquely mapped to any other distribution. Most of the methods for generating independent samples of $X$ involve transformations of random numbers uniformly distributed in the interval $\{0,1\}[\underline{4}, \underline{5}]$. Here, however, for reasons that are evident in what follows, we use a zero mean, unit variance normal distribution as a "seed" to obtain independent samples for a given target distribution. In the following, terms "normal" distribution and "Gaussian" distribution are used interchangeably. The primary goal here is to obtain an algorithm that accurately yields target sample functions that are consistent both with the given distribution function and spectral density (or, equivalently, temporal auto covariance function) of interest. For a large number of samples, $N$, the transformation of Eq. (2.1) accurately yields the distribution of interest. As is shown below, an accurate approximation is obtained for a given target PSD is also obtained from Eq. (2.1) by using a (colored) zero mean, unit variance normally distributed process as a seed whose PSD is equal to the desired target PSD. This Gaussian seed produces signals that have arbitrary means and variances, as implicitly contained in the inverse of the target CDF. Here, we are concerned with the power spectrum of the fluctuations of the random process relative to its mean. As such, the PSD and the corresponding autocovariance are Fourier transform pairs, where the integral of the PSD over all frequencies equals the variance of the process.

In order to obtain sample functions of a normally distributed homogeneous stochastic process with an arbitrary PSD, we use the Fourier transform spectral representation method first introduced by Rice [6] and applied for simulation purposes by Shinozuka and Jan [7] and Shinozuka and Deodatis [8]. In this method, as used here, a discrete Fourier transform of $N$ independent (i.e., white noise) zero mean, unit variance normally distributed temporal sample functions is performed. Denote the sample temporal length by $T$ and the corresponding intersample spacing $t_{S}$, respectively, and we have $T=N t_{S}$. These white noise samples are then "colored" in the Fourier domain by multiplying each of the $j$ th angular frequency components by $\sqrt{2 S\left(\omega_{j}\right) \Delta \omega}$, where $S(\omega)$ is the PSD of interest, $\Delta \omega=\omega_{U} / N$, and $\omega_{U}$ represents an upper cut-off frequency beyond which the PSD may be assumed to be zero for either physical or mathematical reasons. The resulting series is then inverse Fourier transformed back into temporal space. It was shown in [8] that both the autocorrelation function and corresponding PSD of the resulting time series produced by this method rapidly converges as $1 / N^{2}$ to the target autocorrelation function and PSD. Additionally, the process simulated by this technique is ergodic, regardless of the size of $N$. This makes the method directly applicable to a time domain analysis, where the ensemble average and temporal average are equal. Note, regardless of the size of $N$, the results of this procedure remain normally distributed because sums of any number of normal distributions remain normal [9]. Thus, this procedure yields normally distributed sample functions whose PSD and autocovariance function for sufficiently large $N$ are highly accurate representations of the corresponding target functions. We note, in contrast to many previous fast Fourier transform (FFT) algorithms, the number of data supplied to the built-in very efficient and fast Mathematica FFT routine need not have a length equal to a power of 2 . In

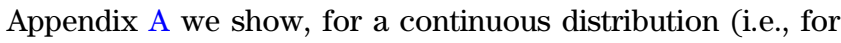
$N \rightarrow \infty$ ), that this Fourier transform spectral representation procedure yields an autocorrelation function that is identical to that of the target.

Yamazaki and Shinozuka developed a method for generating non-Gaussian zero mean multidimensional homogeneous fields [1]. Their method is based on an iterative procedure involving the generation method for zero mean Gaussian homogeneous fields, which have the same variance as the target distribution, on the basis of their spectral representation as discussed above and the mapping technique of Eq. (2.1), which transforms normally distributed sample functions into arbitrary nonnormally distributed sample functions. Yamazaki and Shinozuka claim that, because the transformation of Eq. (2.1) is nonlinear, an iterative procedure must be used in order to obtain good agreement between the simulated and target function's PSDs. These authors then give one numerical example based on a zero mean beta distribution with a Gaussian shaped PSD and find, after three iterations, that the simulated PSD is in good agreement with that of the target. More recently, Minfen et al. [10] proposed an autoregressive (AR) model with order $N$ as the linear system to provide a Gaussian sequence with the desired autocorrelation. A single example of a random sequence with an exponential PDF and an exponential autocorrelation function is given that shows, in their Fig. 2, fairly good simulation results for both the CDF and the autocorrelation function. However, no information is provided as how the order $N$ or the AR model parameters are obtained in this example or, more to the point, in practice, for other target probability distributions. To the best of our knowledge $[\underline{1}, \underline{2}, \underline{10}]$ provide the only numerical examples of the generation of a non-Gaussian distribution with a given target PSD that has appeared in the literature.

In contrast to Yamazaki and Shinozuka's iterative method, for a great majority of cases of interest, we show that it is not necessary to use an iteration scheme; rather, a single application of Eq. (2.1) yields highly accurate results. Apparently, no one has taken the interest to check this out. Additionally, there is no need to assume in Eq. (2.1) zero mean and equal variances for the target and Gaussian distribution, respectively. As an example of the utility of the present method, we consider, as in [1] , the continuous beta distribution with shape parameters $\alpha$ and $\beta$, whose mean and variance are given by $\alpha /(\alpha+\beta)$, and $\alpha \beta /\left[(\alpha+\beta)^{2}(1+\alpha+\beta)\right]$, respectively. The $\mathrm{CDF}$ and corresponding inverse CDF of the beta distribution are given by [3]

$$
\begin{gathered}
F(x)=\text { BetaRegularized }[x, \alpha, \beta], \quad \text { for } 0 \leq x \leq 1, \\
F^{-1}(x)=\text { InverseBetaRegularized }[x, \alpha, \beta], \text { for } 0 \leq x \leq 1 .
\end{gathered}
$$

Two beta distributions with shape parameters $\alpha=4, \beta=2$, and $\alpha=1, \beta=5$ are chosen here in order to consider highly skewed distributions. The PDF of these distributions are shown in Fig. 1(a).

Following Yamazaki and Shinozuka [1], we choose a Gaussian-shaped target PSD of the form 

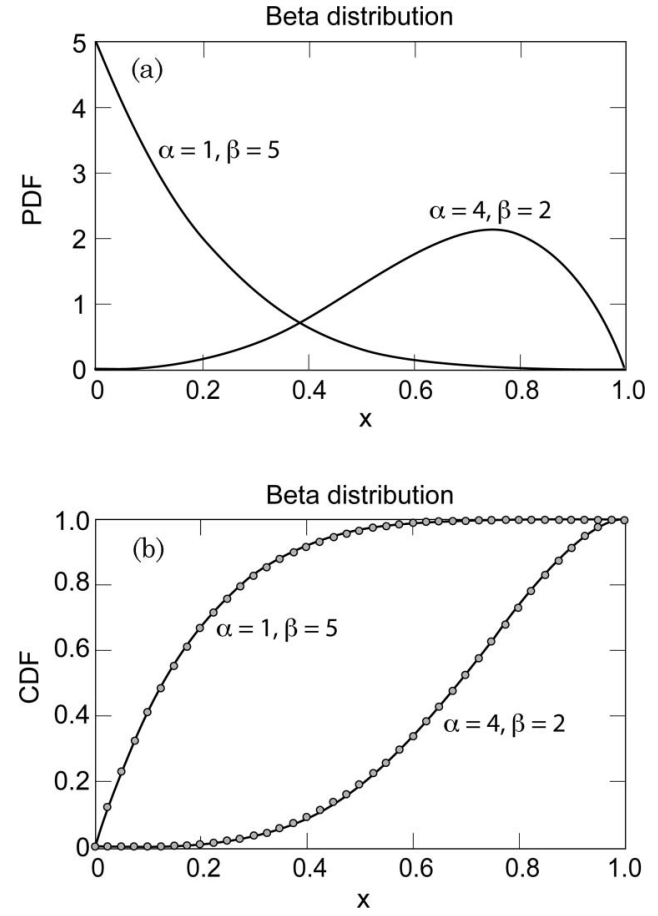

Fig. 1. (a) PDF of the beta distribution as a function of the random variable $x$. (b) Comparison of the CDF of the beta distribution. The simulation [for $N=10^{5}$ sample points and a single application of Eq. (2.1)] and exact results are given by the dotted points, and solid curves, respectively.

$$
S(\omega)=\sigma^{2} \tau_{C} \sqrt{\pi} \exp \left[-\omega^{2} \tau_{C}^{2} / 4\right]
$$

where $\omega$ is the angular frequency, $\sigma^{2}$ is the variance, and $\tau_{C}$ is a measure of the correlation time of the underlying process. Here and in what follows, it is implicitly understood that the variance of the target distribution is identical to the variance obtained by integrating the (illustrative) PSD over all frequencies. Thus, for the PSD of Eq. (2.5) and the beta distribution of Eq. (2.3), we have $\sigma^{2}=\alpha \beta /\left((\alpha+\beta)^{2}(1+\alpha+\beta)\right)$. The simulated colored (dotted points) and exact CDFs (solid curves) are compared in Fig. 1(b). Examination of Fig. 1(b) shows excellent agreement between the simulated and exact results.

The corresponding normalized target temporal correlation coefficient, $R(\tau)$, as obtained from the Wiener-Khintchine transformation, is given by

$$
R(\tau)=\exp \left[-\tau^{2} / \tau_{C}^{2}\right]
$$

Here and in the following, we choose, for convenience, to compare the target and simulated correlation coefficient. Because the correlation coefficient, $R(\tau)$, and the normalized PSD are related via a Fourier transform, agreement between either one of these implies agreement of the other. Figure 2 shows a comparison of the simulated and target correlation coefficient for $\alpha=4, \beta=2$, for $N=10^{5}$ samples, a single application of Eq. (2.1), and, for illustration purposes, we have assumed a normalized correlation time of 10 . Here and in what follows, the normalized time lag is the time lag multiplied by the sample rate. Thus, in this example for a $1 \mathrm{kHz}$ sample rate, the correlation time $\tau_{C}$ equals $10 \mathrm{~ms}$. Examination of Fig. 2 reveals excellent agreement. The corresponding result for

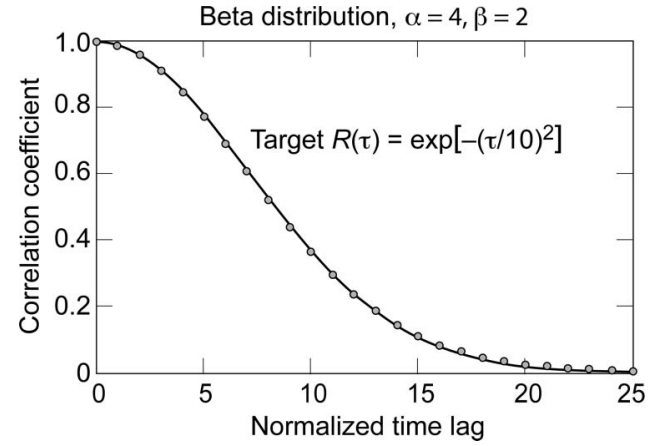

Fig. 2. Simulated and target correlation coefficient as a function of the time lag multiplied by the sample rate for the beta distribution and a single application of Eq. (2.1). The dotted points and solid curve are the simulation and target values, respectively.

$\alpha=1, \beta=5$ is essentially identical to that shown for $\alpha=4$, $\beta=2$ and is not reproduced here. Thus, for this illustration, a single application of Eq. (2.1) yields a sample that is beta distributed with a correlation coefficient/PSD that is an excellent approximation to the target value.

To close this section, we apply the noniterative inverse CDF method to an example PDF and PSD considered in [2] regarding ocean wave surface heights, where the empirical model $\mathrm{PDF}$ is given by [11]

$$
p(x)=\frac{2 \phi^{2}(\kappa)}{(1-\kappa x)^{3}} \exp \left[-\phi^{2}(\kappa)\left(\frac{x}{1-\kappa x}\right)^{2}\right], \quad \text { for } 0 \leq x<1 / \kappa,
$$

where $x=H / H_{\text {rms }}$ is the normalized wave height, $H$ is the wave height, $H_{\mathrm{rms}}$ is the root mean square wave height, $0 \leq \kappa \leq$ 1 is a dimensionless parameter that controls the shape of the wave height distribution [for $\kappa=0$, Eq. (2.7) is the Rayleigh distribution, and for $\kappa \rightarrow 1$ it becomes the Dirac delta distribution], and $\phi(\kappa)=\left(1-\kappa^{0.944}\right)^{1.187}$. The corresponding CDF and inverse CDF are given by

$$
\begin{aligned}
& F(x)=1-\exp \left[-\phi^{2}(\kappa)\left(\frac{x}{1-\kappa x}\right)^{2}\right], \quad \text { for } 0 \leq x<1 / \kappa, \quad(2.8) \\
& F^{-1}(x)=\frac{\phi(\kappa) \sqrt{-\log (1-x)}+\kappa \log (1-x)}{\phi^{2}(\kappa)+\kappa^{2} \log (1-x)}, \quad \text { for } 0 \leq x \leq 1 .
\end{aligned}
$$

The authors of [2] used a shape parameter $\kappa=0.5$ and the Pierson-Moskowitz PSD given by

$$
S_{\mathrm{PM}}(\omega)=\frac{4 \sigma^{2}}{\omega_{N}^{5}} \exp \left(-\omega_{N}^{-4}\right)
$$

where $\omega_{N}=\omega \tau_{C}$ is the normalized angular frequency, $\tau_{C}$ is a measure of the correlation time, and $\sigma^{2}$ is the variance of the process. The corresponding correlation coefficient is obtained from the Wiener-Khintchine relation as

$$
R_{\mathrm{PM}}(\tau)=\sqrt{2 \pi} G_{0,5}^{3,0}\left(\frac{\left(\tau / \tau_{C}\right)^{4}}{256} \mid 0, \frac{1}{2}, 1, \frac{1}{4}, \frac{3}{4}\right)
$$

where $G(\cdot)$ is a Meijer $G$ function [3]. 
Figure $\underline{3}$ shows an example of the resulting time series segment, the simulated colored PDF and CDF, and the corresponding correlation coefficient obtained by using $N=$ $10^{4}$ sample points, $\kappa=0.5$, and $\tau_{C}=10 t_{S}$. Examination of Fig. 3 shows that, even for $10^{4}$ sample points, excellent simulation agreement is obtained from a single application of the inverse CDF method (note, for larger values of $N$, one obtains a much smoother simulated PDF that exhibits much better agreement with the target PDF). In the following, we choose not to display the resulting PDF and CDF comparisons because, for all cases considered, the agreement between simulated and exact results is as least as good as shown in Figs. 2 and 3 . Thus, we obtain, from a single application of Eq. (2.1), a sample time series that is distributed both with a PDF given by Eq. (2.7) and conforms to a correlation coefficient/PSD that is an excellent approximation to the Pierson-Moskowitz target value. Additionally, we note that a single application of the inverse CDF method is not limited to unimodal spectra. To illustrate this, we choose a Rayleigh distribution with a bimodal PSD consisting of two displaced Gaussian-shaped spectra, as illustrated in Fig. 3(e), and the excellent agreement between the corresponding simulated and target correlation coefficient is shown in Fig. 3(f).

\section{ILLUSTRATIVE NUMERICAL EXAMPLES}

In the following we present several illustrative (mathematical) examples of the inverse CDF method applied to stochastic processes primarily of interest to the optics community. In particular, we employ the noniterative inverse CDF method using PSDs of a variety of spectral shapes to illustrate the efficacy of this method. As indicated in Section 2, in what follows, it is implicitly understood that the variance corresponding to the target PDF is identical to the variance obtained by integrating the PSD over all frequencies.

\section{A. Gamma Distribution}

First, we consider the gamma distribution, which has been used extensively in the literature to model the PDF of aperture integrated speckle [12,13]. The CDF and corresponding inverse CDF are given by []ㅡㄹ
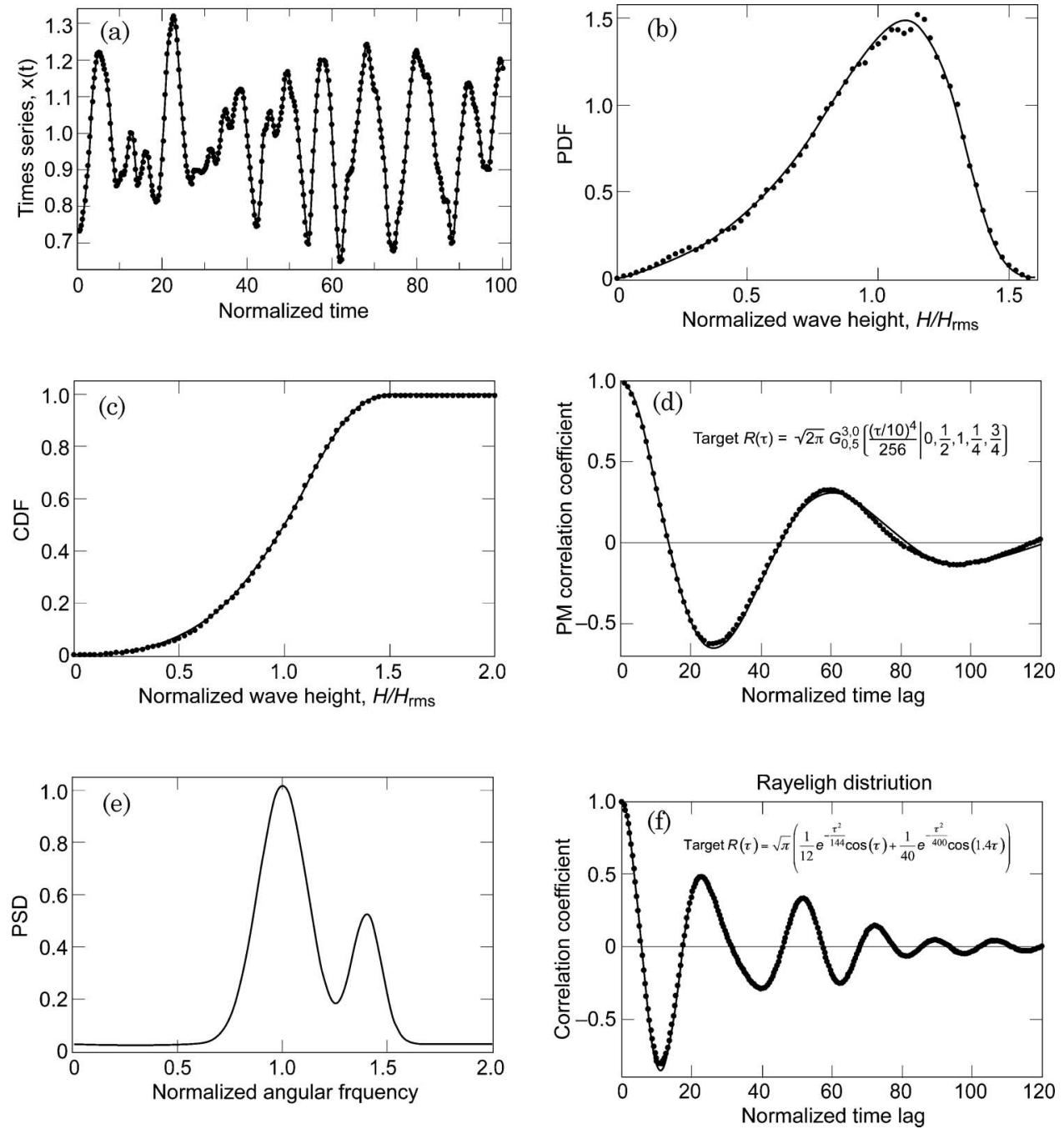

Fig. 3. (a) Simulation of the random process conforming to the PDF given by Eq. (2.7) and the Pierson-Moskowitz PSD for $N=10^{4}$ and $\kappa=0.5$, a time series segment. (b) Same as (a), except here showing the colored PDF. The dotted points and solid curve are the simulation and target values, respectively. (c) Same as (a), except here showing the colored CDF. The dotted points and solid curve are the simulation and target values, respectively. (d) Same as (a), except here showing the Pierson-Moskowitz correlation coefficient. The dotted points and solid curve are the simulation and target values, respectively. (e) Bimodal PSD. (f) Comparison between the simulated and target correlation coefficient of the bimodal PSD shown in (e). 


$$
\begin{aligned}
& \qquad F(x)=1-\frac{\Gamma(m, m x)}{\Gamma(m)}, \text { for } x \geq 0, \\
& F^{-1}(x)=\text { InverseGammaRegularized }[m, 0, m x], \\
& \text { for } 0 \leq x \leq 1,
\end{aligned}
$$

where, for simplicity in notation, we have normalized the level, $x$, to its mean, and $\Gamma(m, m x)$ is the incomplete gamma function. The parameter $m$ can be physically interpreted as the mean number of speckles contained within a collecting aperture [12]. Two correlation functions commonly appearing in measurements related to speckle phenomena are the Gaussian, given by Eq. (2.4), used in speckle correlation [14] and the narrowband filtered signal appropriate for spatial filtering velocimetry [15] given by

$$
R_{\mathrm{NB}}(\tau)=\cos \left(\omega_{0} \tau\right) \exp \left[-\tau^{2} / \tau_{C}^{2}\right],
$$

where $\omega_{0}$ is the center (angular) frequency of the narrowband filter. In Figs. $\underline{4}$ and $\underline{5}$, the simulated and target correlation coefficients are plotted as functions of normalized time lag for $m=1$ (i.e., the exponential PDF) and 6 for a normalized correlation time of 10. Examination of these figures reveals that, for $m=1$, good agreement is obtained between the simulated and the target correlation coefficients (e.g., for the Gaussian shape, a least square fit for the "correlation time" yields a value of 9.54), while, for larger values of $m$, the agreement becomes excellent. In Appendix B we present an estimate for various PDFs of interest where the transformation of Eq. (2.1) is expected to yield simulation results for the correlation coefficients that are good approximations to the corresponding target value. In particular, for the gamma distribution, highly accurate results are expected for $m>1$, which is in accord with the results shown in Figs. 4 and 5.

\section{B. Weibull Distribution}

For simplicity in notation, we consider the Weibull distribution with shape parameter $k$ and denote by $x$ the random variable normalized to the shape parameter. The Weibull distribution is used for describing the distribution of wind velocities at various altitudes relevant for lidar systems. The CDF and inverse $\mathrm{CDF}$ of this distribution are given by

$$
\begin{gathered}
F(x)=1-\exp \left[-x^{k}\right], \quad \text { for } x \geq 0, \\
F^{-1}(x)=(-\log [1-x])^{1 / k}, \quad \text { for } 0 \leq x \leq 1,
\end{gathered}
$$

respectively, where $x \geq 0$ and $k$ can be any real positive number. Note, for $k=1$ and 2, the Weibull distribution becomes the exponential and the Rayleigh distribution, respectively. To illustrate the effectiveness of a single application of the inverse CDF method, we choose another class of target correlation coefficients rather than using the same ones used in Subsection 3.A (where essentially identical results are obtained). Here we choose a class of correlation coefficients given by

$$
R(\tau)=\frac{1}{2^{\nu-1} \Gamma(\nu)}\left(\tau / \tau_{C}\right)^{\nu} K_{\nu}\left(\tau / \tau_{C}\right), \quad \text { for } \nu>1 / 2,
$$
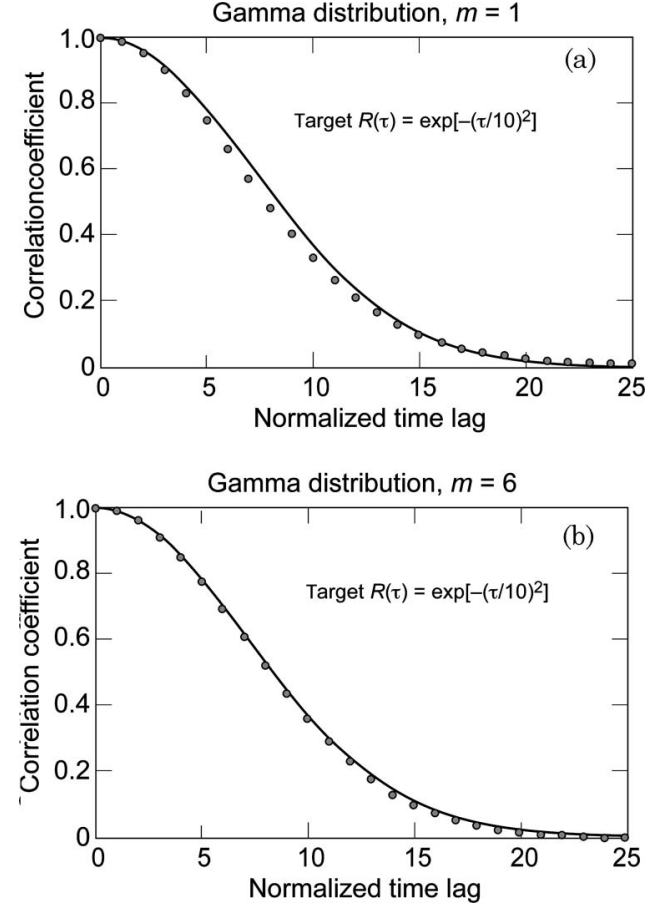

Fig. 4. (a) Simulated and target correlation coefficient as a function of the time lag for the gamma distribution for $m=1$, a Gaussianshaped correlation coefficient, and a single application of Eq. (2.1). The dotted points and solid curve are the simulation and target values, respectively. (b) Same as (a), except that $m=6$.

where $\tau_{C}$ is a measure of the correlation time and $K_{\nu}(\cdot)$ denotes the modified Bessel function of the second kind of order $\nu$. Real stationary processes are characterized by having
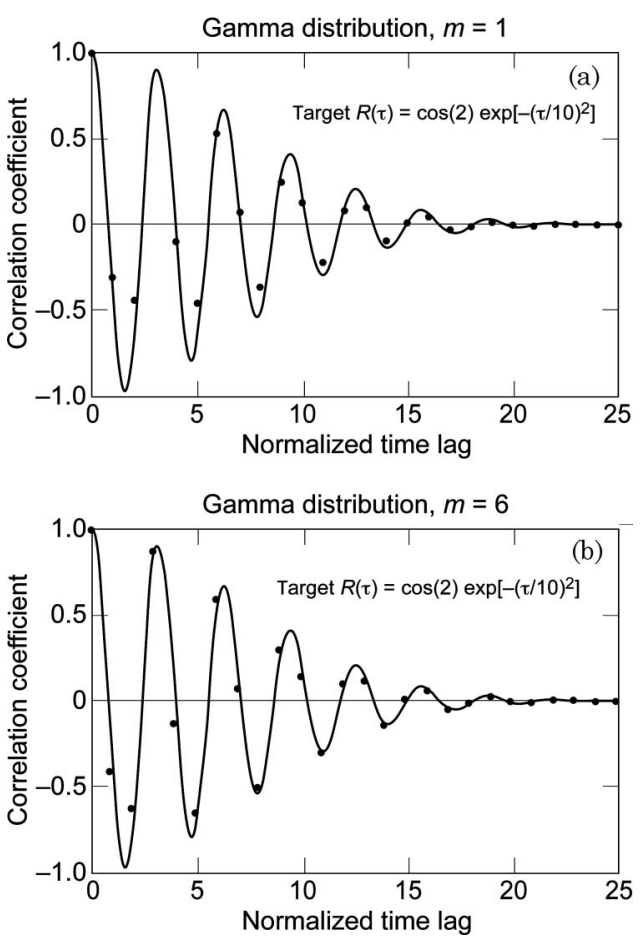

Fig. 5. (a) Simulated and target correlation coefficient as a function of the time lag for the gamma distribution for $m=1$, a narrowband filtered correlation coefficient, and a single application of Eq. (2.1). The dotted points and solid curve are the simulation and target values, respectively. (b) Same as (a), except that $m=2$. 
correlation functions with the property that their first and second derivative evaluated at zero time lag equals zero and is negative, respectively [9], and this is obtained for the correlation coefficients of Eq. (3.6) for any real number $\nu>1 / 2$ [16]. The corresponding PSD, normalized to the variance of the process, is given by

$$
S(\omega)=\frac{\sqrt{\pi} \Gamma(\nu+1 / 2)}{\Gamma(\nu)\left(1+\omega^{2} \tau_{C}^{2}\right)^{\nu+1 / 2}} .
$$

Weibull distributions with a shape parameter $k$ between about 1.4 and 2.3 have been used to model wind velocity distributions [17]. For illustrative purposes, we choose a shape parameter $k=1.8$ and a correlation coefficient given by Eq. (3.6) for $\nu=3 / 5$. The simulation results of a single application of Eq. (2.1) are shown in Fig. 6, where very good agreement with the target correlation coefficient is obtained. For larger values of both $k$ and/or $\nu$, even better results are obtained. As indicated in Appendix B, for the Weibull distribution, good agreement between the simulated and target correlation coefficient is obtained for $k>1$ by a single application of Eq. (2.1); this has been confirmed by many numerical simulations, which are not reproduced here.

Finally, in Fig. 7, we present a simulated time series corresponding to this example, where we have assumed a $1 \mathrm{kHz}$ sampling rate and a correlation time of $10 \mathrm{~ms}$.

\section{Power Law Distribution}

The CDF and inverse CDF of the power law distribution can be expressed as

$$
\begin{gathered}
F(x)=x^{m}, \quad \text { for } 0 \leq x \leq 1, \\
F^{-1}(x)=x^{1 / m}, \quad \text { for } 0 \leq x \leq 1,
\end{gathered}
$$

where $x$ denotes a suitably normalized random variable and the parameter $m$ can be any real number greater than zero. For example, such a distribution describes the irradiance statistics for a Gaussian-shaped laser beam in the presence of line-of-sight mechanical platform jitter, where $x$ is the irradiance normalized to its peak value (i.e., on-axis value), and $m$ is given by the square of the ratio of the $1 / \sqrt{e}$ angular beam radius to the $1-\sigma$, single axis standard deviation of residual jitter [18]. A range of $m$ values of interest is between $1 / 3$ and 2, where the corresponding Strehl ratio due to residual jitter is in

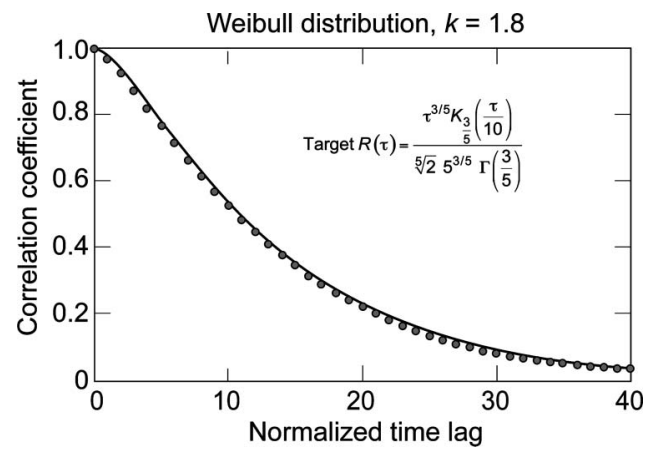

Fig. 6. (a) Simulated and target correlation coefficient as a function of time lag for the Weibull distribution for $k=1.8$, and a single application of Eq. (2.1). The dotted points and solid curve are the simulation and target values, respectively.

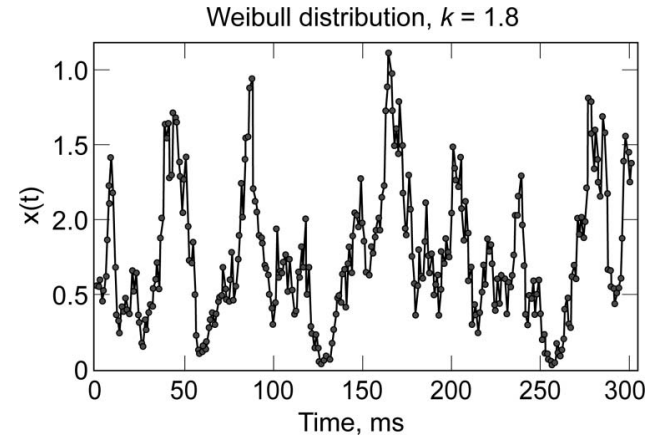

Fig. 7. Example of a simulated time series corresponding to the Weibull distribution for a shape parameter of 1.8 and the correlation coefficient of Eq. (3.6) for $\nu=3 / 5$. For this example, we have assumed a sample rate of $1 \mathrm{kHz}$ and a correlation time $\tau_{C}=10 \mathrm{~ms}$.

the range of $0.25-0.67$. For $m>$ than about 0.67 , the Strehl ratio approaches unity and simulations to mimic system behavior are not necessarily needed. In Figs. 8(a) and 8(b), we compare the results for the simulated correlation coefficient for the power law distribution for $m=1 / 3$ and 2, respectively, for an illustrative example target correlation coefficient shape given by Eq. (3.6) for $\nu=5 / 6$. Examination of Figs. 8(a) and $8(\mathrm{~b})$ reveals fair and very good agreement for $m=\overline{1 / 3}$ and 2 , respectively. These features are consistent with the results of Appendix $\underline{B}$ for the power law distribution, where good agreement between the simulated and target correlation coefficient is obtained for $m>1$ with a single application of Eq. (2.1).

\section{Lognormal Distribution}

The lognormal distribution for the irradiance distribution is applicable for laser propagation through the atmosphere
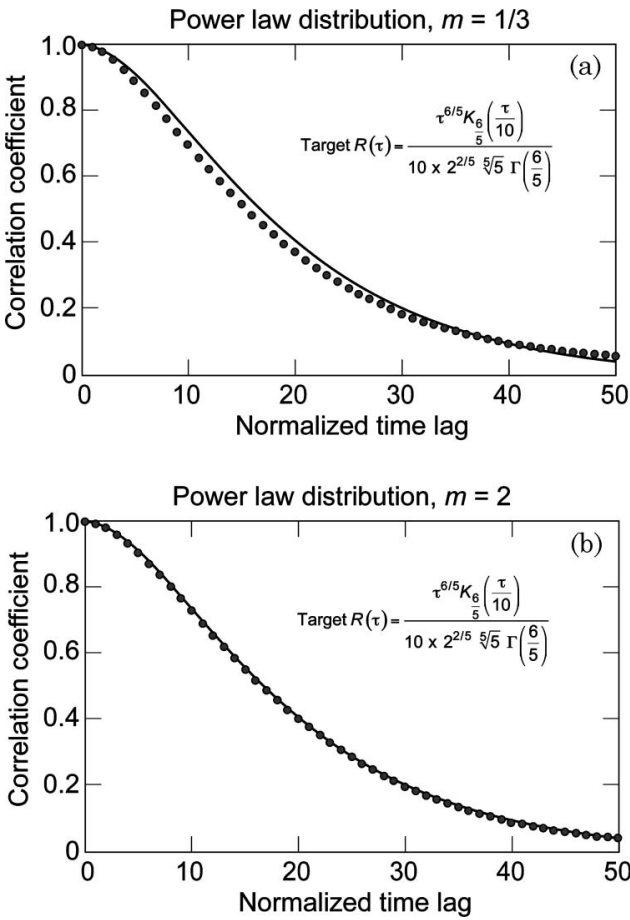

Fig. 8. (a) Simulated and target correlation coefficient as a function of time lag for the power law distribution for $m=1 / 3$, and a single application of Eq. (2.1). The dotted points and solid curve are the simulation and target values, respectively. (b) Same as (a), except that $m=2$. 
under weak scintillation conditions [19]. As an illustrative example, we consider the optical irradiance statistics associated with ground-based stellar observations at modest elevation angles. Under conditions where the log-intensity variance $\sigma_{\ln I}^{2} \ll 1$, the lognormal CDF, and corresponding inverse CDF can be expressed as

$$
\begin{gathered}
F(x)=\frac{1}{2}\left(1+\operatorname{erf}\left[\frac{\log x+\sigma_{\ln I}^{2} / 2}{\sqrt{2} \sigma_{\ln I}}\right]\right), \quad \text { for } x \geq 0, \\
F^{-1}(x)=\exp \left[x \sigma_{\ln I}-\sigma_{\ln I}^{2} / 2\right], \quad \text { for } 0 \leq x \leq 1,
\end{gathered}
$$

$x$ denotes the irradiance level normalized to its mean value. For weak scintillation conditions, assuming Taylor's hypothesis, Tatarskii has shown that the temporal intensity correlation coefficient and corresponding PSD are given by [20]

$$
\begin{aligned}
& R(\tau)=3.864 \operatorname{Im}\left[e^{11 \pi i / 12}{ }_{1} F_{1}\left(-\frac{11}{6} ; 1 ; \frac{i \tau^{2}}{4}\right)\right]-2.3724 \tau^{5 / 3}, \\
& S(\omega)=\frac{2.577}{\omega^{8 / 3}}\left(1-\frac{8 \Gamma(17 / 6)}{11 \Gamma(7 / 3) \omega^{2}} \times \operatorname{Im}\left[e^{i \omega^{2}} U(\omega)\right]\right)
\end{aligned}
$$

where

$$
\begin{aligned}
U(\omega)= & \frac{\pi}{\sin (\pi b)}\left(\frac{{ }_{1} F_{1}\left(a ; b ;-i \omega^{2}\right)}{\Gamma(b) \Gamma(a-b+1)}\right. \\
& \left.-\frac{\left(-i \omega^{2}\right)^{1-b}{ }_{1} F_{1}\left(a-b+1 ; 2-b ;-i \omega^{2}\right)}{\Gamma(a) \Gamma(2-b)}\right) .
\end{aligned}
$$
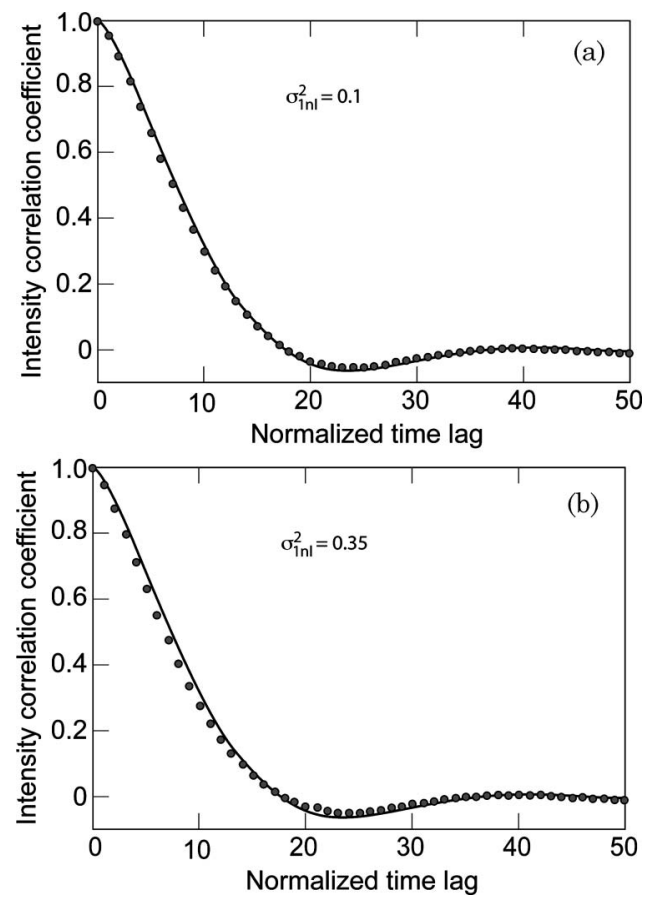

Fig. 9. (a) Simulated and target correlation coefficient of Eq. (3.12) as a function of time lag for the lognormal distribution for $\sigma_{\ln I}^{2}=0.1$, and a single application of Eq. (2.1). The dotted points and solid curve are the simulation and target values, respectively. (b) Same as (a), except that $\sigma_{\ln I}^{2}=0.35$.
Im denotes the imaginary part, $a=1 / 2, b=-4 / 3$, and ${ }_{1} F_{1}(\cdot)$ is the Kummer confluent hypergeometric function. In Figs. 9(a) and $9(\mathrm{~b})$, we compare the results for the simulated correlation coefficient for the lognormal distribution for $\sigma_{\ln I}^{2}=0.1$ and 0.35 , respectively. As these examples illustrate, a single application of Eq. (2.1) yields very good agreement between the simulated and target correlation coefficients for weak scintillation conditions, and indicates that, for these conditions, the inverse CDF method is a viable simulation tool. For values of the log-intensity variance approaching unity, the agreement of the simulations gracefully degrades but never distorts in shape. These results are in agreement with those presented in Appendix B, where good agreement is expected for values of the log-intensity variance less than unity.

\section{E. Rice-Nakagami Distribution}

As a final example, we illustrate the utility of obtaining the mean level crossing rate for a PDF whose corresponding CDF cannot be obtained analytically [21]. The PDF and CDF of the Rice-Nakagami is given by

$$
\begin{gathered}
p(x)=2 x \exp \left[-\left(x^{2}+C^{2}\right)\right] I_{0}(2 x C), \quad \text { for } x \geq 0, \\
F(x)=\int_{0}^{x} 2 y \exp \left[-\left(y^{2}+C^{2}\right)\right] I_{0}(2 y C) \mathrm{d} y, \quad \text { for } 0 \leq x \leq 1,
\end{gathered}
$$

where $I_{0}(\cdot)$ is the modified Bessel function of the first kind of order zero, $C$ is a real constant, and both $x$ and $C$ are normalized to the "square root" of twice the variance of the underlying normal distribution [9]. In a variety of applications, the Rice-Nakagami distribution is used to model the intensity distribution in a speckle pattern that consists of a specular component and a diffuse scattered component [22]. To obtain a colored Rice-Nakagami sample distribution, we proceed as follows. First, for a given value of $C$, a tabulated set of data values in the form $\left\{F\left(x_{i}\right), x_{i}\right\}$ over a suitable range of $x$ values is obtained via numerical integration of Eq. (3.16). Next, for the Rice-Nakagami distribution, an accurate nonlinear fit of this "data set" to a model inverse CDF function of the form $F_{\mathrm{RN}}^{-1}(x)=a x^{n}+a_{1} x+a_{3} x^{3}+a_{5} x^{5}+b \log (1-x)$, where $a, a_{1}, a_{3}, a_{5}$, and $b$ are the model fit parameters to be determined, is made. Finally, this analytic model is then used in Eq. (2.1) to obtain the suitable colored Rice-Nakagami sample distribution. We choose $C=1 / 2$ to illustrate the efficacy of this method. Figure 10 shows the excellent comparison of the inverse CDF (dotted points) obtained numerically to the analytic model (solid curve) obtained via least squares fit. Figure 11 is the corresponding comparison of the simulation results based on a Gaussian-shaped PSD to the target value. Examination of Fig. 11 reveals excellent agreement between the target and simulation correlation coefficient. Similar results have been obtained both for a wide range of values of $C$ and target PSD. Thus we conclude that a single application of Eq. (2.1) is sufficient to obtain accurate colored simulation results for the Rice-Nakagami distribution.

\section{DISCUSSION AND CONCLUSION}

A simple and efficient method has been derived for simulating time or space series with a given probability distribution 
function and a desired spectral content. The method has been tested on a series of signals, of which the major part was related to issues within the field of optics. A convincing agreement between the desired and the obtained parameters for the signals has been observed. Although not discussed, the formalism is easily applicable for two-dimensional applications. Thus, precise and fast simulations of the response of optically based sensor systems can be performed for digitally derived speckle patterns with spectral contents of practical concern. The latter may be important for simulation of optical vortices [23], in the finite size effects in speckles arising from scattering off rough surfaces [24], or for determination of speckle statistics for non-Gaussian speckle fields [25]. Recently, the issue of speckle reduction has gained importance, as laserbased displays are assumed to enter the market in the near future $[26,27]$. In the case that moving diffusers facilitate the task of speckle reduction, the intensity probability distribution function will be highly non-Gaussian [28]. For optically based sensors, and especially sensors based on the use of coherent radiation, the probability distribution function will depend highly on the scattering properties of the interrogated medium, on the optical train between the object and the detector system, and on the detector system itself. In some cases, analytical expressions for the simulated signal may even be derived, thus further reducing the effort in analyzing the performance of the optical system.

In summary, the method presented here provides an efficient tool for simulating signals with both a given probability distribution function with an arbitrary mean value and a spec-

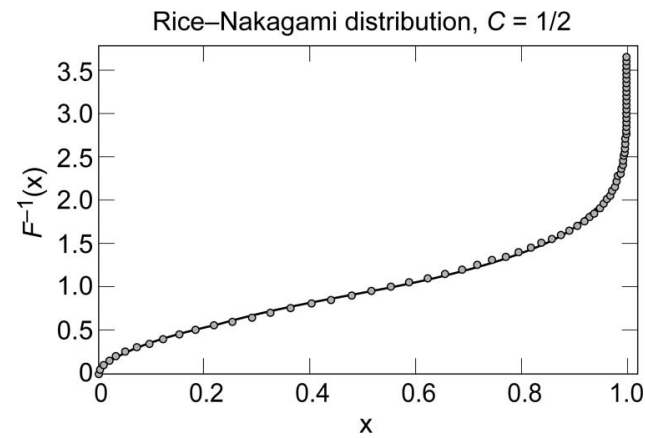

Fig. 10. Comparison of the Rice-Nakagami inverse CDF for $C=1 / 2$ obtained via numerical integration (dotted points) to the analytic model obtained by a least square fit (solid curve).

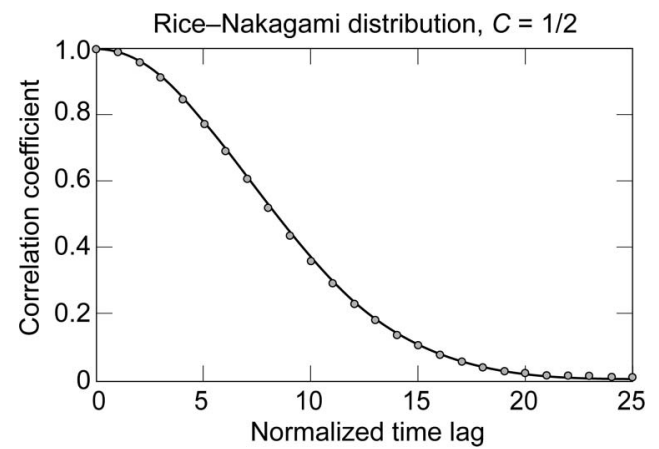

Fig. 11. Simulated and target Gaussian correlation coefficient as a function of time lag for the Rice-Nakagami distribution for $C=1 / 2$, and a single application of Eq. (2.1). The dotted points and solid curve are the simulation and target values, respectively. tral content, which is a close approximation to the desired one with very little distortion in shape. Because, in practice, when dealing with random processes, there is always some uncertainty as to the parameter values involved, and, as a result, the inverse CDF method may be sufficient for the simulation system response. However, if higher accuracy is desired, one can then utilize the iterative procedure outlined in [1]. A number of other probability distributions and PSDs have been simulated with equally satisfactory results, as shown in Section 3 (e.g., the chi square distribution, for cases where the degree of freedom exceeds unity). Finally, we note that two signals with identical PDF and PSD might have different higher-order moments that may influence the outcome in a measurement setup. As a result, careful application of the present method should be conducted, especially when the result depends on moments of the signal exceeding second order. If higher order moments are vital, an investigation of a possible difference between the "desired" and the "resulting" moment of a given order should be conducted. In the present work, we have demonstrated our simulation method focusing on stationary random processes. However, nothing precludes the $\mathrm{CDF}$ in Eq. (2.1) from being a function of time. Specifically, one could employ Eq. (2.1) with a CDF whose parameters (e.g., the CDF's variance) are time-varying functions. Then, at every instant of time one could employ Eq. (2.1) to generate a random variable from the time-varying CDF. In this way, the method could be extended to non-stationary random processes.

\section{APPENDIX A}

Consider a real stationary continuous white noise zero mean, unit variance normal random process, $Y(t)$, whose Fourier transform is given by

$$
\tilde{Y}(\omega)=\int_{-\infty}^{\infty} \mathrm{d} t Y(t) \exp (i \omega t) .
$$

Next, consider the quantity $\tilde{G}(\omega)$ defined as

$$
\tilde{G}(\omega)=\int_{-\infty}^{\infty} \mathrm{d} t \sqrt{S(\omega)} Y(t) \exp (i \omega t),
$$

where $S(\omega)$ is the target PSD. The inverse Fourier transform of $\tilde{G}(\omega)$ is then

$$
G(t)=\int_{-\infty}^{\infty} \mathrm{d} \omega \tilde{G}(\omega) \exp (-i \omega t) / 2 \pi
$$

Because integrals are essentially infinite summations, and $Y(t)$ is a Gaussian process, the quantity $G(t)$ is also a Gaussian process. We now show that the autocovariance function of $G(t)$ is given by the Fourier transform of the PSD. The autocovariance function, $B_{G}(\tau)$, of $G(t)$ is given by

$$
\begin{aligned}
B_{G}(\tau) & =\left\langle G(t) G^{*}(t+\tau)\right\rangle \\
& =\int_{-\infty}^{\infty} \mathrm{d} t_{1} \int_{-\infty}^{\infty} \mathrm{d} t_{2} \int_{-\infty}^{\infty} \mathrm{d} \omega_{1} \int_{-\infty}^{\infty} \mathrm{d} \omega_{2} \sqrt{S\left(\omega_{1}\right) S\left(\omega_{2}\right)}\left\langle Y\left(t_{1}\right) Y\left(t_{2}\right)\right\rangle \\
& \times \exp \left[-i \omega_{1}\left(t_{1}-t\right)+i \omega_{2}\left(t_{2}-t-\tau\right)\right] /(2 \pi)^{2} .
\end{aligned}
$$

Because $Y(t)$ is a unit variance white noise Gaussian process, we have $\left\langle Y\left(t_{1}\right) Y\left(t_{2}\right)\right\rangle=\delta\left(t_{1}-t_{2}\right)$, where $\delta(\cdot)$ is the Dirac delta function, and Eq. (A4) becomes 


$$
\begin{aligned}
B_{G}(\tau)= & \int_{-\infty}^{\infty} \mathrm{d} t_{1} \int_{-\infty}^{\infty} \mathrm{d} \omega_{1} \int_{-\infty}^{\infty} \mathrm{d} \omega_{2} \sqrt{S\left(\omega_{1}\right) S\left(\omega_{2}\right)} \\
& \times \exp \left[-i \omega_{1}\left(t_{1}-t\right)+i \omega_{2}\left(t_{1}-t-\tau\right)\right] /(2 \pi)^{2} \\
= & \int_{-\infty}^{\infty} \mathrm{d} \omega_{1} \int_{-\infty}^{\infty} \mathrm{d} \omega_{2} 2 \pi \delta\left(\omega_{1}-\omega_{2}\right) \sqrt{S\left(\omega_{1}\right) S\left(\omega_{2}\right)} \\
& \times \exp \left[i \omega_{1} t-i \omega_{2}(t+\tau)\right] /(2 \pi)^{2} \\
= & \int_{-\infty}^{\infty} \mathrm{d} \omega S(\omega) \exp [-i \omega \tau] / 2 \pi
\end{aligned}
$$

Thus, $G(t)$ is a Gaussian process colored with the given target PSD.

\section{APPENDIX B}

To ascertain the parameter regimes where a single application of Eq. (2.1) yields a satisfactory approximation to the target correlation coefficient, we proceed as follows.

We have

$$
Z(t)=F^{-1}\left[F_{G}(t)\right]
$$

where, for the zero mean, unit variance normally distributed seed,

$$
F_{G}(t)=\frac{1}{2}\left[1+\operatorname{erf}\left(\frac{x(t)}{\sqrt{2}}\right)\right] .
$$

Expanding $Z(t)$ in a Taylor series about the mean value of $x(t)$ (i.e., $x=0$ ) yields

$$
Z(t)=\sum_{n=0}^{\infty} \frac{x^{n}(t)}{n !} \frac{d^{n}}{d x^{n}}\left[F^{-1}\left[F_{G}(x)\right]\right]_{x=0}
$$

The temporal autocorrelation function of $Z$ is then given by

$$
\begin{aligned}
B(\tau)= & \langle Z(t+\tau) Z(t)\rangle=\sum_{n=0}^{\infty} \sum_{m=0}^{\infty} \frac{\left\langle x^{n}(t+\tau) x^{m}(t)\right\rangle}{n ! m !} \\
& \times\left(\frac{d^{n}}{d x^{n}}\left[F^{-1}\left[F_{G}(x)\right]\right]_{x=0}\right) \\
& \times\left(\frac{d^{m}}{d x^{m}}\left[F^{-1}\left[F_{G}(x)\right]\right]_{x=0}\right),
\end{aligned}
$$

where we have tacitly assumed that the random process $Z$ is ergodic, and angular brackets denote the ensemble/temporal average. For a zero mean, unit variance normally distributed random process, it can shown that [29]

$$
B_{G}(\tau)=\left\langle x^{n}(t+\tau) x^{m}(t)\right\rangle=i^{(n+m)} \frac{\partial^{n}}{\partial \omega_{1}^{n}} \frac{\partial^{m}}{\partial \omega_{2}^{m}} \chi\left(\omega_{1}, \omega_{2}\right) \mid \begin{aligned}
& \mid \begin{array}{l}
\omega_{1}=0 \\
\omega_{2}=0
\end{array},
\end{aligned}
$$

where the corresponding characteristic function, $\chi\left(\omega_{1}, \omega_{2}\right)$, is given by

$$
\chi\left(\omega_{1}, \omega_{2}\right)=\exp \left[-\frac{\omega_{1}^{2}+\omega_{2}^{2}}{2}-\omega_{1} \omega_{2} R_{G}(\tau)\right]
$$

and $R_{G}(\tau)$ is the Gaussian seed correlation coefficient, which is used in the text as the target value.

The correlation coefficient, $R(\tau)$, of $Z$ is given by

$$
R(\tau)=\frac{\langle Z(t+\tau) Z(t)\rangle-\langle Z\rangle^{2}}{\left\langle Z^{2}\right\rangle-\langle Z\rangle^{2}}
$$

To obtain a first-order estimate of the parameter range where $R$ is satisfactorily approximated by the target correlation function $R_{G}$, we find to terms of second order in $R_{G}$ that

$$
R(\tau)=R_{G}(\tau) \psi
$$

where

$$
\psi=\frac{4 \pi\left[F^{-1}(1 / 2)\right]^{\prime}+R_{G}\left[F^{-1}(1 / 2)\right]^{\prime \prime}}{4 \pi\left[F^{-1}(1 / 2)\right]^{\prime}+\left[F^{-1}(1 / 2)\right]^{\prime \prime}} .
$$

The correction factor given by Eq. (B9) is used here as a rule of thumb to obtain the parameter range where a single application of Eq. (2.1) yields satisfactory results. Examination of Eq. (B8) reveals that, for $\psi$ near unity, the transformed correlation coefficient $R$ is well approximated by the input "target" correlation coefficient $R_{G}$. To obtain numerical results, we arbitrarily use a value of $R_{G}=1 / e$, and, in Fig. 12 , we plot $\psi$ as a function of the corresponding free parameter in the gamma, power law, and Weibull distributions, while the corresponding results for the lognormal distribution is plotted in Fig. 13. As indicated in Section 3, the results shown in Figs. 12 and $\underline{13}$ are in accord with corresponding simulation results. Other target distributions and PSDs can be treated accordingly.

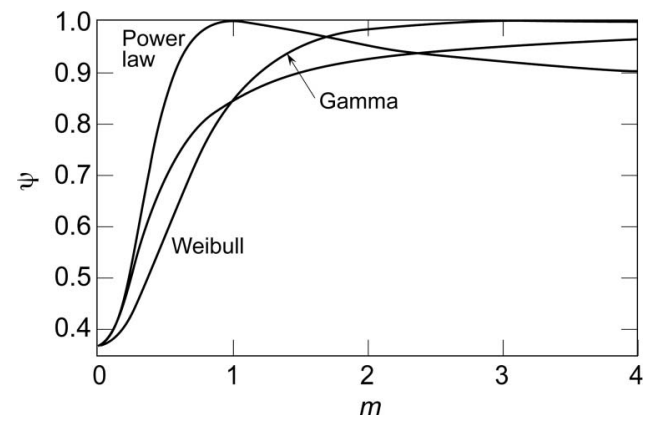

Fig. 12. Correction factor $\psi$ as a function of the parameter $m$. Note that, for brevity in notation, we have here denoted the shape parameter $k$ for the Weibull distribution by $m$.

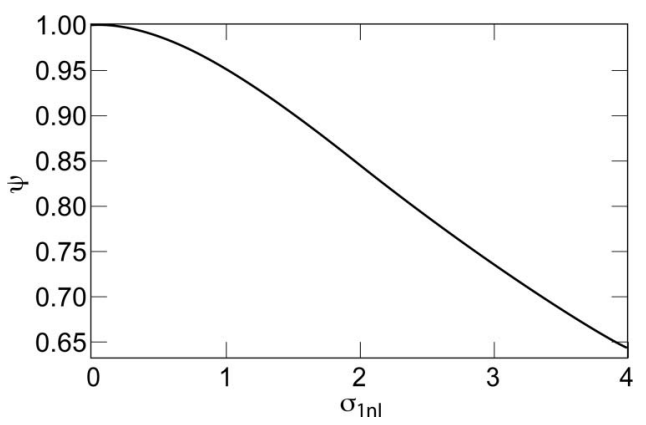

Fig. 13. Correction factor $\psi$ for the lognormal distribution as a function of the parameter $\sigma_{\ln I}$. 


\section{REFERENCES AND NOTES}

1. F. Yamazaki and M. Shinozuka, "Digital generation of nonGaussian stochastic fields,” J. Eng. Mech. Div., Am. Soc. Civ. Eng. 114, 1183-1197 (1988)

2. J. M. Nichols, C. C. Olson, J. V. Michalowitz, and F. Bucholtz, "A simple algorithm for generating spectrally colored non-Gaussian signals," Prob. Eng. Mech. 25, 315-322 (2010).

3. Wolfram Mathematica, Version 7 (Cambridge University, 2008).

4. P. Bratley, B. L. Fox, and L. E. Schrage, A Guide to Simulation, 2nd ed. (Springer-Verlag, 1987).

5. R. W. Rubinstein, Simulation and the Monte Carlo Method (Wiley, 1981)

6. S. O. Rice, "Mathematical analysis of random noise," in Selected Papers on Noise and Stochastic Processes (Dover, 1954).

7. M. Shinozuka and C. M. Jan, "Digital simulation of random processes and its application," J. Sound Vib. 25, 111-128 (1972).

8. M. Shinozuka and G. Deodatis, "Simulation of stochastic processes by spectral representation," Appl. Mech. Rev. 44, 191-204 (1991)

9. P. Beckmann, Probability in Communication Engineering (Harcourt, Brace \& World, 1967), Section 6.6.

10. S. Minfen, F. H. Y. Chan, and P. J. Beadle, "A method for generating non-Gaussian noise series with specified probability distribution and power spectrum," in ISCAS '03. Proceedings of the 2003 International Symposium on Circuits and Systems (IEEE, 2003), Vol. 2, II-1-II-4.

11. J. Mendez and S. Castanedo, "A probability distribution for depth-limited extreme wave heights in a sea state," Coastal Eng. 54, 878-882 (2007).

12. J. W. Goodman, Speckle Phenomena in Optics: Theory and Applications (Roberts, 2006).

13. R. Barakat, "Level-crossing statistics of aperture averagedintegrated isotropic speckle," J. Opt. Soc. Am. A 5, 1244-1247 (1988).

14. U. Schnell, J. Piot, and R. Dändliker, "Detection of movement with laser speckle patterns: statistical properties," J. Opt. Soc. Am. A 15, 207-216 (1998).

15. Y. Aizu and T. Asakura, Spatial Filtering Velocimetry, Fundamentals and Applications, Vol. 116 of Springer Ser. Opt. Sci. (Springer-Verlag, 2006).

16. In this regard we note, for $\nu=1 / 2$, Eq. (3.6) becomes an exponential, whose first and second derivative evaluated at the origin are -1 and 1 , respectively, and thus cannot be representative of a real physical process.

17. S. C. Pryor, M. Nielsen, R. J. Barthelmie, and J. Mann, "Can satellite sampling of off shore wind speeds realistically represent wind speed distributions? Part II: Quantifying uncertainties associated with distribution fitting methods," J. Appl. Meteorol. Climatol. 43, 739-750 (2004).

18. H. T. Yura, "LADAR detection statistics in the presence of pointing errors," Appl. Opt. 33, 6482-6498 (1994).

19. L. C. Andrews, R. L. Phillips, and C. Y. Hopen, Laser Beam Scintillation with Applications (SPIE, 2001), Chap. 2.

20. V. I. Tatarskii, The Effects of the Turbulent Atmosphere on Wave Propagation (Israel Program for Scientific Translations, 1971).

21. The CDF of the Rice-Nakagami distribution can be expressed in terms of a Marcum $Q$ function, which are tabulated, but not supported, to the best of our knowledge, by any commercial commuter programs such as Mathematica and MATLAB.

22. J. W. Goodman, Speckle Phenomena in Optics: Theory and Applications, (Roberts, 2006), Section 3.2.2.

23. D. V. Kiesewetter, "Numerical simulation of a speckle pattern formed by radiation of optical vortices in a multimode optical fibre," Quantum Electron. 38, 172-180 (2008).

24. A. Garcia-Martin, R. Gomez-Medina, J. J. Saenz, and M. Nieto-Vesperinas, "Finite-size effects in the spatial distribution of the intensity reflected from disordered media," Phys Rev. B 62, 9386-9389 (2000).

25. J. W. Goodman, "Speckle with a finite number of steps," Appl. Opt. 47, A111-A118 (2008).

26. H. Satoh, K. Sekiya, T. Kawakami, Y. Kuratomi, B. Katagiri, Y. Suzuki, and T. Uchida, "On the effect of small moving diffusers to the speckle reduction in laser projection displays," in IDW '09-Proceedings of the 16th International Display Workshops (2009), pp. 1361-1364.

27. P. Lehmann, A. Schone, and J. Peters, "Non-Gaussian far field fluctuations in laser light scattered by a random phase screen," Optik 95, 63-72 (1993).

28. J. W. Goodman, Speckle Phenomena in Optics: Theory and Applications (Roberts, 2006), Section 3.6.

29. M. Shinozuka and G. Deodatis, "Simulation of stochastic processes by spectral representation,” Appl. Mech. Rev. 44, 191-204 (1991) 